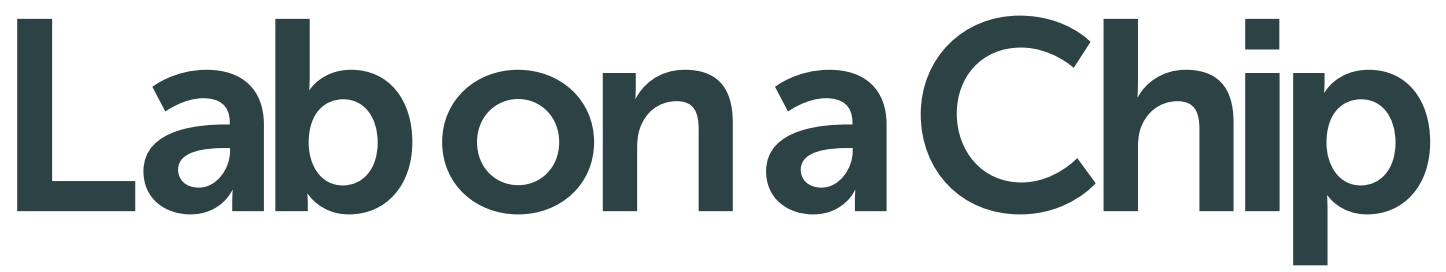

Miniaturisation for chemistry, physics, biology, materials science and bioengineering rsc.li/loc

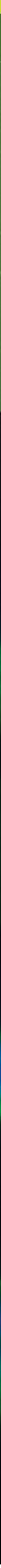

ISSN 1473-0197

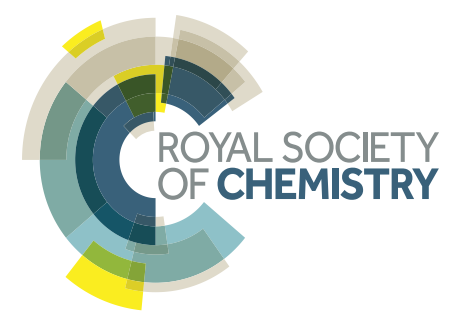




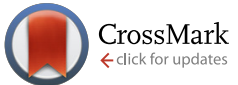

Cite this: Lab Chip, 2017, 17, 629

Received 24th October 2016, Accepted 15th January 2017

DOI: 10.1039/c6lc01319h

\section{A simple and low-cost chip bonding solution for high pressure, high temperature and biological applications $\dagger$}

\author{
M. Serra, ${ }^{a b c}$ I. Pereiro, $\star^{* a b c}$ A. Yamada, ${ }^{a b c}$ J.-L. Viovy, ${ }^{a b c}$ \\ S. Descroix ${ }^{a b c}$ and D. Ferraro ${ }^{a b c}$
}

rsc.li/loc

The sealing of microfluidic devices remains a complex and timeconsuming process requiring specific equipment and protocols: a universal method is thus highly desirable. We propose here the use of a commercially available sealing tape as a robust, versatile, reversible solution, compatible with cell and molecular biology protocols, and requiring only the application of manually achievable pressures. The performance of the seal was tested with regards to the most commonly used chip materials. For most materials, the bonding resisted 5 bars at room temperature and 1 bar at $95{ }^{\circ} \mathrm{C}$. This method should find numerous uses, ranging from fast prototyping in the laboratory to implementation in low technology environments or industrial production.

\section{Introduction}

Most microfluidic applications rely on the efficient bonding of two different parts to create enclosed channels. However, this fabrication step is still often complex, time-consuming and represents a factor that limits the spread of lab-on-a-chip systems in non-technical environments. A variety of techniques have been proposed over the years for the bonding of microfluidic chips fabricated with different materials and, in general, all these methods require either specific surface chemistries, high temperature, extremely clean surfaces or specialized tools. ${ }^{1}$ For example, polydimethylsiloxane (PDMS), the most widely used material by the microfluidic community, is most often bonded to a glass slide or to another PDMS layer by previous oxygen plasma ${ }^{2}$ or corona discharge ${ }^{3}$ activation. Alternatively, techniques based on chemical modifications have been proposed. ${ }^{4-6}$ Thermoplastics, on the other hand, are often sealed by applying pressure and temperature

\footnotetext{
${ }^{a}$ Laboratoire Physico Chimie Curie, Institut Curie, PSL Research University, CNRS UMR168, 75005, Paris, France. E-mail: iago.pereiro@curie.fr

${ }^{b}$ Sorbonne Universités, UMPC Univ. Paris 06, 75005 Paris, France

${ }^{c}$ Institut Pierre-Gilles de Gennes, 75005, Paris, France

$\dagger$ Electronic supplementary information (ESI) available. See DOI: 10.1039/ c6lc01319h

\$ Equal contribution.
}

either above their glass transition temperature $\left(T_{\mathrm{g}}\right)$ by thermal bonding or below $T_{\mathrm{g}}$ by a solvent assisted approach, ${ }^{7,8}$ although recently the possibility of bonding cyclic olefin copolymer (COC) by applying pressure at room temperature has been reported with the right mixture of solvents. ${ }^{9}$ These approaches require a delicate tuning to avoid the deformation of the initial structures, especially if they are small or present a high aspect ratio. Then, glass to glass bonding requires chemical procedures at high temperature ${ }^{10}$ or highly corrosive acids. ${ }^{11}$ Furthermore, in principle, 3D printing allows the production of directly closed devices, but open microfluidic channels are often preferred to easily remove the support material or the uncrosslinked resin, especially when high printing resolutions are needed. ${ }^{12}$ Therefore, the bonding strategy for $3 \mathrm{D}$ printed devices must be customized depending on the microfabrication strategy and on the resin material.

Alternative approaches have been reported, notably with the use of pre-polymerized adhesive layers to obtain a more versatile bonding. For example, UV curing adhesives (e.g. NOA 81$)^{13,14}$ have been reported as a possible solution to allow patterning directly in the adhesive followed by its bonding by sticking at room temperature; however, homogeneous thickness, patterning and possible channel clogging remain a challenge. Thin layers of SU-8 have also been proposed as a bonding material, allowing a more precise patterning, but needing high temperature or UV activation to obtain good bond strengths. ${ }^{1,15}$ Furthermore, the use of a PDMS prepolymer film as an adhesive layer has been shown for PDMS/PDMS, glass/glass and PDMS/glass bonding. ${ }^{16}$ This method has also been extended for the bonding of PDMS devices to photoresist, metallic ${ }^{17}$ and plastic substrates. ${ }^{18}$

Microfabrication based on pressure sensitive adhesive (PSA) has also been reported for the fast and easy prototyping of entire microfluidic devices manufactured by desktop craft cutters. ${ }^{19,20}$ Some reported works combine this type of adhesive with more commonly employed materials. In particular, double-coated adhesive tape has been proposed for the fabrication of PDMS-tape composites that can be bound to other 
materials, ${ }^{21}$ but this still implies elaborate fabrication protocols. More recently, it was suggested that another type of adhesive tape could be directly used to seal PDMS devices, ${ }^{22}$ however it required long baking time (around $2 \mathrm{~h}$ ) to obtain a resistant bonding, and it is still limited to working pressures below 0.5-1 bars. Beyond this pressure limit, SaffmanTaylor fingers appear around the inlet region. Furthermore, the resistance to high or cycling temperature was not investigated, and bio-compatibility tests were limited to confined droplets, limiting the possible applications of the resulting devices.

In this work, we propose the use of a commercially available PCR adhesive tape, based on the PSA concept, as a multi-purpose bonding solution for microfluidic channels patterned in a wide variety of materials commonly used by the microfluidic community, i.e. PDMS, COC, poly(methylmethacrylate) (PMMA), polycarbonate (PC), glass and stereolithographic 3D printer resin. We demonstrate that this PCR tape sealing is able to sustain high pressures (up to $5 \mathrm{bar}$ ) directly after bonding without any further strengthening steps, even working at relatively high temperatures ( $\left.\mathrm{ca} .100{ }^{\circ} \mathrm{C}\right)$. The deformation of the tape under high pressure was characterized, and its impact on high-frequency droplet generation was investigated, proving that the bonding covers the required pressure range without any instability. Furthermore, we explored the biocompatibility of the tape for cell culture and molecular biology. In particular, its relatively inert surface allowed successful cell culture and on-chip PCR, whereas its transparency enabled high-resolution optical microscopy observations. Finally, this tape bonding strategy also presents an easy reversible bonding, making expensive to fabricate or difficult to clean microfluidic devices readily available for reuse.

\section{Results}

A straight channel $(200 \mu \mathrm{m}$ in width and depth, $15 \mathrm{~mm}$ in length) was patterned in PDMS (Dow Corning, Sylgard 184 $10: 1$ ratio, by replica molding of a brass micromilled mold), COC (Topas 6013, by hot embossing of the same mold), PMMA (OTMT), PC (Radiospares) (by direct material micromilling), glass slide (ROTH, with laser engraver C180II from GCC LaserPro) and stereolithographic 3D printer resin (DS2000, by DWS). The PCR sealing tape (ThermalSeal RTS $^{\text {TM }}$, Excel Scientific) having a thickness of $100 \mu \mathrm{m}$, a contact angle of $101.9 \pm 1.1^{\circ}$ and a roughness of $\mathrm{Ra}=131.9 \pm 3.4$ $\mathrm{nm}$ (see ESI $\dagger$ Fig. S1a), was cut with a size of $20 \times 35 \mathrm{~mm}$ and manually placed over the pattern, pressing directly with fingers or by simple tweezers. We estimated the local force applied manually to be between 1 and $5 \mathrm{~kg}$, corresponding to 1-5 bars by direct finger pressing (surface of $\sim 1 \mathrm{~cm}^{2}$ ) or 1050 bars with a tweezer tip (surface of $\sim 1 \mathrm{~mm}^{2}$ ). However, for a user-independent characterization of the bond strength, the protocol was performed by applying pressure with a hydraulic press at 3 bar, corresponding to the pressure range applied by hand. The bond strength was then evaluated by filling the chip with water and progressively increasing the inlet pressure of 100 mbar every 30 seconds with a constantly closed outlet (pressure controller MFCS, Fluigent). The maximum pressures obtained before the bond burst are shown in Fig. 1 for either a room temperature environment $\left(c a .25^{\circ} \mathrm{C}\right)$ or when placed on a heating plate (Techne Prime) at $95{ }^{\circ} \mathrm{C}$, equipped with a flat adapter. ${ }^{23}$ In the latter case, in order to evaluate the effect of the pressure applied during the bonding, we also tested 20 bar (equivalent to local pressures applicable by a tweezer tip).

As observed, at room temperature the bonding with all thermoplastics and 3D resin resisted at pressures over 5 bar (which represents the highest pressure that we could apply), while PDMS and glass presented maximum bursting pressures of 4.0 and 2.6 bar, respectively. In any case, this is comparable to the bond strengths that can be obtained by oxygen plasma for PDMS-PDMS (1.4-3.4 bar) or PDMS-glass (2.7-4.8 bar). ${ }^{2}$

The same tests were repeated under high temperature conditions $\left(95{ }^{\circ} \mathrm{C}\right)$, resulting in a decrease in the bursting pressures of all the materials, but which should be nonetheless sufficient for most microfluidic applications (1.6-1.8 bar for all thermoplastics and PDMS). Additionally, we observed that it is possible to increase the bond strength for most materials by increasing the bonding pressure ( 20 bar), allowing maximum pressures over 2 bar for PDMS and PMMA and even beyond 3 bar in the case of COC (see Fig. 1). The lower values obtained in the case of the $3 \mathrm{D}$ printed device at $95{ }^{\circ} \mathrm{C}$ might be due to the nature of the printed material itself, which is originally designed for the production of transparent medical prostheses with working temperatures between $20^{\circ} \mathrm{C}$ and $60{ }^{\circ} \mathrm{C}$ (DigitalWAX DL-260).

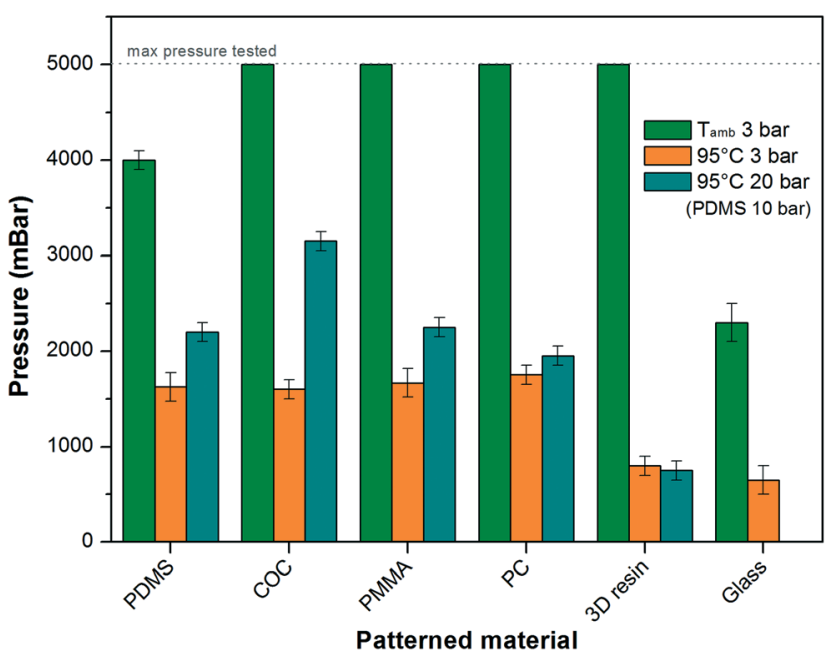

Fig. 1 Bond strength of the PCR adhesive tape for channels patterned in different materials. The pressures at which a bonding burst occurred are shown for each material for different fabrications bonding (pressure at 3,10 or 20 bar) and experimental conditions (room temperature or $95{ }^{\circ} \mathrm{C}$ ). The highest tested pressure was 5 bar. An enhanced adhesion at higher bonding pressure (10 or 20 bar) is observed for $95^{\circ} \mathrm{C}$ tests. Every test has been repeated 3 times $(n=3)$. 
It is important to underline that the bonding obtained with any of the tested pressures can be very easily removed with manual pulling forces (e.g. with tweezers), leaving no visible trace of the adhesive. The device can then be immediately resealed for further use or for the liquid content collection.

Since COC is a widely used thermoplastic material in microfluidics due to its remarkable properties, such as compatibility with biological applications, ${ }^{24,25}$ chemical resistance ${ }^{26,27}$ and high transparency, ${ }^{28}$ we selected it for forthcoming evaluations.

The integrity and stability of the bonding was further evaluated by confocal microscopy (TCS SP8, Leica) by filling COC channels of different widths $(200,500$, and $1000 \mu \mathrm{m})$ and same depth $(200 \mu \mathrm{m})$ with a fluorescein solution $(3 \mu \mathrm{M}$ in water, by Sigma-Aldrich) and applying pressure during the measurement. This allowed us to evaluate the total area of the channel section in a wide range of pressures as indicated in Fig. 2a, where the initial area is normalized as 1. As shown, the smallest channel presents the highest stability, with an increase in the total area of the section below 11\% for an applied pressure of 3 bar. For larger widths the section increase is proportionally higher, although still below $10 \%$ for pressures up to 1.5 bar for $500 \mu \mathrm{m}$ wide channels and up to 0.5 bar for $1 \mathrm{~mm}$. Therefore, the maximum pressure applicable must be chosen considering the geometry of the microfluidic channels. However, for most microfluidic applications in which working pressures are below 1 bar and channel sizes in the range of tens to hundreds of microns, this adhesive tape solution seems to be well adapted. Furthermore, confocal imaging shows that the wall formed by the adhesive tape tends to stay flat for all pressures and the deformation takes place only at the interface between the tape and the chip material (Fig. 2b). Unbonding of this interface is the source of rupture if the pressure is increased to the maximum values shown in Fig. 1. We hypothesize that this might explain the

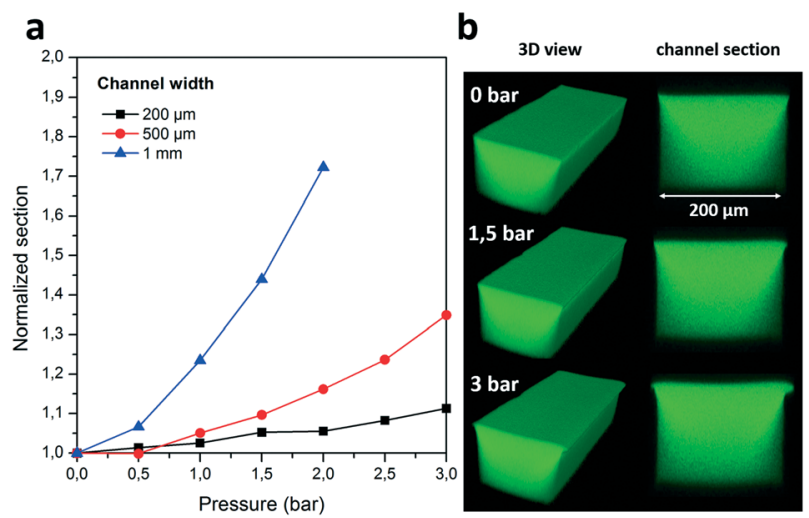

Fig. 2 (a) Confocal microscopy measurements were used to evaluate the total section of three tape-sealed channels of different widths for a range of pressures. (b) A 3D reconstruction of confocal microscopy images and relative cross sections of the $200 \mu \mathrm{m}$-wide channel closed from the top at different applied pressures; during the pressure application the squared cross section is well preserved, with a slight deformation on the borders at high pressures. lower bond strength observed for glass, as the aqueous solution could have an easier access to the interface due to higher wettability of the material. However, in all cases the aspect ratio of the section is highly preserved even in the upper pressure range. Further, any possible deformation at high pressure is homogeneously distributed along the length of the channel, as seen in Fig. 2b, avoiding the formation of Saffman-Taylor fingers that would implicate the formation of dead-end channels and stagnant areas. Finally, we evaluated the reversibility of this deformation by performing pressure cycles in which increasing pressures were alternated with zero pressure conditions (for the $200 \mu \mathrm{m}$-wide channel). The deformation was seen to be partly reversible, with a permanent section increase below $5 \%$ for pressures up to 2 bars (see ESI $\dagger$ Fig. S2).

In order to evaluate the geometrical stability over a long experimental time with a particularly pressure-demanding application, ${ }^{29}$ tests were performed by high-frequency generation of monodisperse water droplets in oil (FC-40 oil, 3M) containing 1\% (w/w) Krytox-PEG surfactant (Fluoro-Surfactant, by Ran Biotechnologies). In detail, the COC device was designed with a flow focusing geometrical configuration sealed with the adhesive (lower insert of Fig. 3a: oil and water inlets $200 \mu \mathrm{m}$ wide, droplet generation channel $100 \mu \mathrm{m}$ wide and $50 \mu \mathrm{m}$ high). Since the droplet size depends on the channel geometry, at first, it was constantly evaluated for $25 \mathrm{mi}$ nutes during the generation of either 1300 (inlet oil flow rate $2.4 \mu \mathrm{L} \mathrm{s}^{-1}$ and water $0.4 \mu \mathrm{L} \mathrm{s}^{-1}$, flows set by Cetoni syringe pumps) or 4500 droplets per second (inlet oil flow rate $6 \mu \mathrm{L}$ $\mathrm{s}^{-1}$, water $0.9 \mu \mathrm{L} \mathrm{s}^{-1}$ ) by their recording at a frequency of 7400

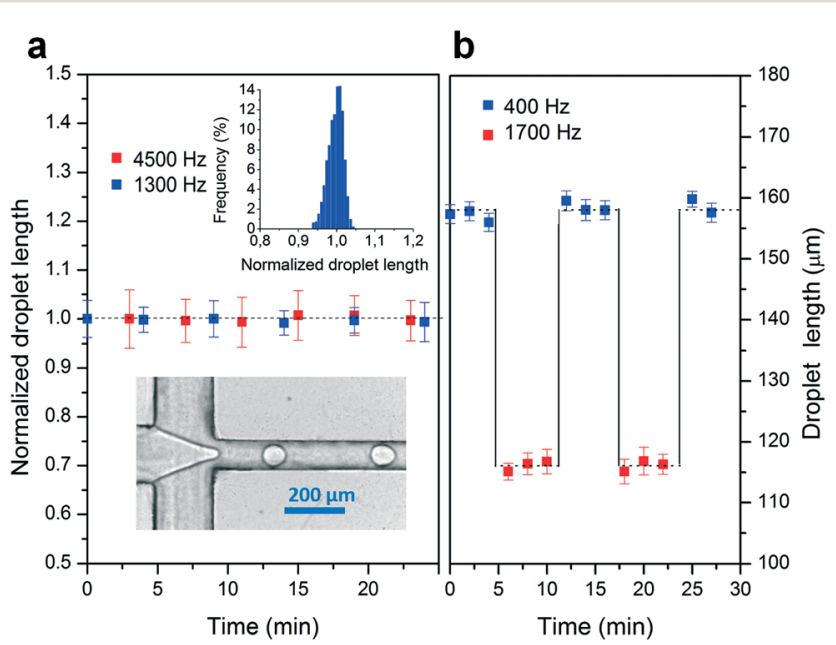

Fig. 3 (a) Normalized droplet length as a function of time for a droplet generation by flow focusing $(n=3)$ at $1300 \mathrm{~Hz}$ (blue dots, CV $<2.8 \%$ ) and $4500 \mathrm{~Hz}$ (red dots, coefficient of variation (CV) $<4.8 \%$ ) presenting a Gaussian distribution as shown in the upper insert (for $1300 \mathrm{~Hz}$ ). Droplet lengths are normalized by the length of the first droplet acquired. The channel geometry is shown in the lower insert. (b) Droplets were generated between two different frequencies (400 and $1700 \mathrm{~Hz}$, blue and red dots respectively) by an interval change in the flow rate of the aqueous phase: oil flow rate $4 \mu \mathrm{L} \mathrm{s} \mathrm{s}^{-1}$ and water between $0.4 \mu \mathrm{L} \mathrm{s}^{-1}$ (red dots) and $0.8 \mu \mathrm{L} \mathrm{s}^{-1}$ (blue dots). Droplet size was equally conserved under sequential cyclic stresses as a function of time. 
$\mathrm{Hz}$ (Nikon Ti microscope, Phantom v4.2 camera by Vision Research) followed by image processing (user-customized LabView program). These frequencies correspond to a range of pressures in the generation channel between 0.4 and 1 bar, covering the range of values for the inlet pressure typically used for droplet generation by flow-focusing. ${ }^{30}$ As shown in Fig. 3a, the droplet size was highly stable and reproducible during all the experimental time, confirming the absence of any deformation in the geometry of the channel. Furthermore, this stability is maintained even in the case of longer experimental times, as indicated in ESI $\dagger$ Fig. S3. The results show in all cases a Gaussian distribution (Fig. 3a upper insert) of the droplet size with a relatively low coefficient of variation below $2.8 \%$ at $1300 \mathrm{~Hz}$ and below $4.8 \%$ at $4500 \mathrm{~Hz}$, indicating again a highly preserved geometry. Next, the stability of the bonding was further tested with pressure cycles in which the flow rate of the aqueous phase was periodically switched between two values keeping a constant oil flow rate $\left(4 \mu \mathrm{L} \mathrm{s}^{-1}\right)$. The two frequency values tested (400 and $1700 \mathrm{~Hz}$ ) were chosen to represent the typical working conditions for flow focusing, in a range between 500 and $2000 \mathrm{~Hz} \cdot{ }^{30,31}$ This resulted in monodisperse droplets generated at two different frequencies $\left(400 \mathrm{~Hz}\right.$ water at $0.4 \mu \mathrm{L} \mathrm{s}^{-1}$ and $1700 \mathrm{~Hz}$ water at $0.8 \mu \mathrm{L} \mathrm{s}^{-1}$ ) and two different sizes that showed an equally high stability over time. In particular, we do not observe neither deviations in the droplet length due to plastic deformation of the device, nor any slow transient behavior that would suggest elastic deformation of the adhesive tape after each change in experimental conditions.

The chemical resistance of the sealing and adhesive in the presence of organic solvents was also evaluated by sealing glass channels containing the chemical solution (see ESI $\dagger$ Fig. S4 for details). The integrity was not visibly affected by the presence of polar solvents (ethanol, dimethyl sulfoxide, acetonitrile), although it was compromised by non-polar solvents (toluene, cyclohexane) after 24 hours of evaluation periods. We further investigated the possible use of the adhesive for microchip capillary electrophoresis applications. In this case a straight COC channel having a cross section of $200 \times 200 \mu \mathrm{m}$ and a length of $3 \mathrm{~cm}$ was sealed with the adhesive, and the electroosmotic mobility $\left(\mu_{\text {eo }}\right)$ inside was obtained according to the current monitoring method ${ }^{32}$ in the presence of phosphate buffer pH 7.5. A HVS448 $1500 \mathrm{~V}$ power supply (LabSmith, Livermore, CA) was employed to provide high voltage and current measurements. A cathodic electroosmotic flow was observed, and the obtained values $\left(\mu_{\text {eo }}=1.69 \times 10^{-4} \pm 0.29 \mathrm{~cm}^{2} \mathrm{~V}^{-1} \mathrm{~s}^{-1}\right)$ were comparable to the case of whole COC chips $\left(\mu_{\text {eo }}=2.76 \times 10^{-4} \pm 0.14 \mathrm{~cm}^{2} \mathrm{~V}^{-1}\right.$ $\left.\mathrm{s}^{-1}\right),{ }^{33}$ evidencing the absence of a dramatic impact of the adhesive tape regarding electrokinetic behavior.

Finally, the tape solution proposed here has been tested for compatibility with both cell culture and molecular biology. The former was performed by closing a PDMS chamber $(9 \times 2 \mathrm{~mm}, 60 \mu \mathrm{m}$ high, $1.2 \mu \mathrm{L}$ internal volume, connected to four external well reservoirs, see ESI $\uparrow$ Fig. S5) with either a standard glass slide or the adhesive tape. PDMS was chosen here because of its gas permeability, which is crucial for cell culture. Both chips were oxygen plasma treated for $30 \mathrm{sec}$ onds, filled with phosphate buffered saline (PBS) containing $0.01 \mathrm{mg} \mathrm{mL} \mathrm{mL}^{-1}$ poly-D-lysine (Sigma-Aldrich) for promoting the cell adhesion, and incubated for 2 hours at room temperature. The chambers were sequentially rinsed with PBS and with cell culture medium (DMEM GlutaMax high glucose supplemented with $10 \%$ fetal bovine serum (v/v), Thermo Fisher Scientific). Next, $3 \mu \mathrm{L}$ of medium containing MadinDarby canine kidney (MDCK) cells at $6 \times 10^{5}$ cells per mL was injected in the chips prior to $10 \mathrm{~min}$ incubation in a humidified $\mathrm{CO}_{2}$ incubator $\left(37^{\circ} \mathrm{C}, 5 \% \mathrm{CO}_{2}\right)$ for cell attachment to the surface (adhesive tape or glass). After filling each reservoir with $50 \mu \mathrm{L}$ of culture medium, the chips were enclosed in a plastic box containing $1 \mathrm{mg} \mathrm{mL}^{-1}$ ethylenediaminetetraacetic acid (EDTA, Sigma-Aldrich) to avoid evaporation and placed in the incubator for the following days. Phase-contrast images of cell growth were taken after 24, 48 and $72 \mathrm{~h}$ as shown in Fig. 4a. As observed, the cells presented an apparently equivalent adhesion to the surface of both glass and the adhesive tape, resulting in comparable cell growth rates and spreading. Full confluency was reached at day 4; at this point the cells had been fixed by incubation with $4 \%$ paraformaldehyde (Electron Microscopy Sciences) in PBS for $10 \mathrm{~min}$, permeabilized by incubation with $0.2 \%$ Triton-X (Life Technologies) in PBS for $30 \mathrm{~min}$, and stained for nucleus and actin by incubation with $1 \mu \mathrm{g} \mathrm{mL}^{-1}$ Hoechst 33258 (Sigma-Aldrich) and 1/300 volume of Alexa Fluor® 647 Phalloidin (Thermo Fisher Scientific) in PBS, respectively, for $2 \mathrm{~h}$ at room temperature. Fig. $4 \mathrm{~b}$ shows a fluorescence image (Nikon Ti Microscope, CoolSNAP HQ2) of the cells grown on the surface of the adhesive, presenting a healthy configuration. Furthermore, this not only shows the possibility of cell culture on the adhesive tape opportunely treated, but also the ability to perform high quality fluorescence imaging through it with no loss of quality, auto-fluorescence artifacts or light absorption (see ESI $\dagger$ Fig. S1b for the UV-visible absorption spectrum of the tape).

The compatibility of the method with molecular biology applications in which the reagent solution is in direct contact with the adhesive was then tested. An on-chip PCR was performed to verify the stability of the adhesive for a high number of fast temperature cycles as well as the viability of the biological protocol. A solution containing the template DNA molecules ( $3 \mathrm{ng} \mu \mathrm{L}^{-1}, 2500 \mathrm{bp}$ ), enzymes (Phusion HighFidelity DNA polymerase, ThermoFischer), dNTP mix and PCR primers were prepared and loaded inside a tape-sealed COC chip containing a straight channel $(300 \mu \mathrm{m}$ width, 300 $\mu \mathrm{m}$ depth) and a middle circular chamber ( $6 \mathrm{~mm}$ diameter, same depth) with a total volume of $15 \mu \mathrm{L}$. The PCR cycle parameters were: denaturation $98^{\circ} \mathrm{C} 30 \mathrm{~s}$, annealing $58^{\circ} \mathrm{C} 10 \mathrm{~s}$ and extension $72{ }^{\circ} \mathrm{C} 30 \mathrm{~s}$ for a total of 35 cycles. The solution was recovered at the end of the process by pipetting and migrated in an agarose gel (SYBR(R) Green I stain) together with the products of a negative control performed with no initial DNA template, following the same protocol. The results are 

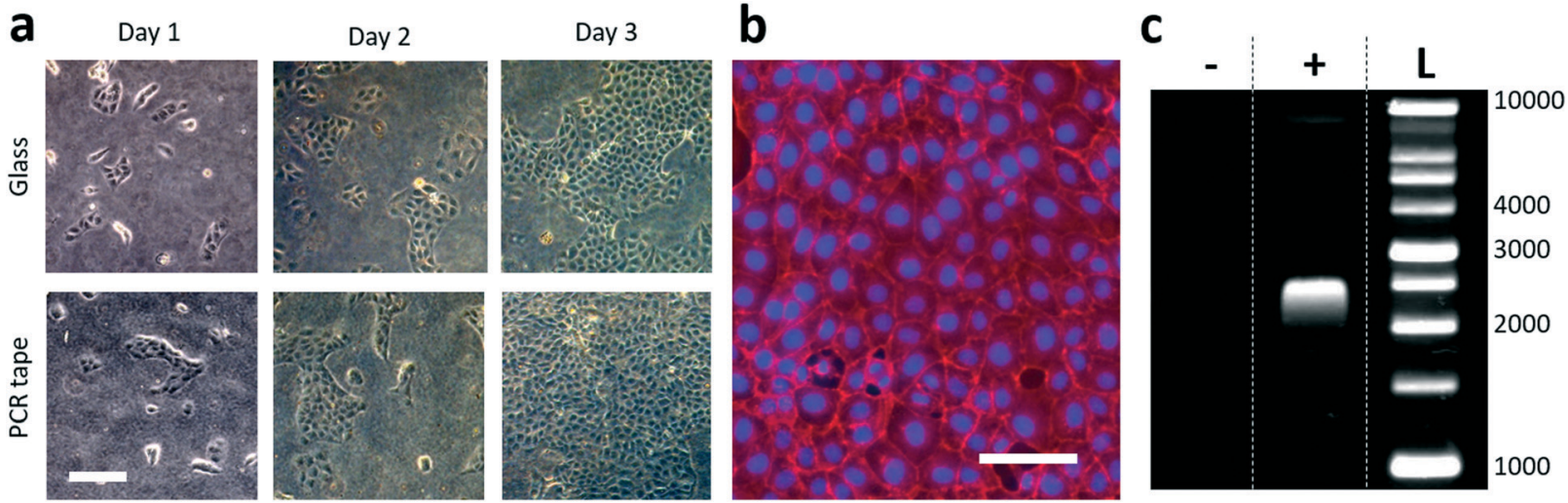

Fig. 4 (a) Phase contrast images of MDCK cells during three days of culture in a PDMS chip sealed either with glass or the adhesive tape (scale bar: $200 \mu \mathrm{m}$ ). (b) Cell fixation and fluorescein staining was performed after cell confluency showing cell nuclei (blue) and actin filaments (red) (scale bar: $100 \mu \mathrm{m}$ ). (c) DNA products recovered from an on-chip PCR in a tape-sealed chamber and visualized by agarose gel electrophoresis. -: Negative control (no DNA target); +: positive control (1 ng of DNA target); L: DNA ladder.

shown in Fig. 4c. The presence of PCR products, as indicated by the obtained positive bands, confirm the viability of enzymatic reactions with high temperature cycling and with the reagents in direct contact with the adhesive tape.

\section{Conclusions}

The PCR adhesive tape evaluated in these tests shows remarkable properties for the easy, low cost and reversible bonding of chips made with a wide range of transparent materials commonly used in the microfluidics community. Besides requiring no heating, a strong bonding can be obtained with the local forces typically obtained with a manual pressing. This is therefore an interesting alternative to more costly and complex bonding procedures that can only be performed in clean room-like facilities, making it suitable and convenient even for educational purposes. On the other hand, as demonstrated by the experiments performed with a press, the technique can also be implemented in manufacturing processes, and in this case manual bonding can facilitate prototyping and is not a mandatory step. The bonding remains highly resistant even for high temperature applications $\left(95{ }^{\circ} \mathrm{C}\right.$ ), allowing in particular PCR cycling procedures that can lead to important vapor pressures inside the microfluidic channels. Moreover, the aspect ratio of the channel section is highly preserved, which, coupled to the low surface roughness of the adhesive, allows precise and demanding microfluidic procedures such as monodisperse droplet generation. Its optical properties in terms of transparency and low autofluorescence are also particularly interesting to include a window with high optical quality, into devices prepared by printed resins that usually present a low optical transmittance and/or a rough surface, as well as for general biological imaging. These optical properties should make possible the high quality imaging of biological processes such as cell culture or enzymatic reactions, which can be performed in direct contact with the surface of the tape. This is further reinforced by the fact that the flat configuration of the adhesive is well preserved even under pressure conditions. Finally, the bonding reversibility allows an easy adhesive removal and the possibility to reuse the chip after rinsing or to collect the sample inside (e.g. cultured cells) for further analysis. Therefore, we believe that this adhesive tape could be a versatile and simple bonding solution for a wide range of common microfluidic applications in both specialized and non-technical environments.

\section{Acknowledgements}

This work is supported by the French National Research Agency (ANR) as part of the "Investissements d'Avenir" program (reference: ANR 10-NANO 0207), ARC foundation for young researcher fellowship (D. F.) and ERC Advanced Grant CellO (FP7-IDEAS-ERC-321107). MS was supported by a Curie Institute International PhD fellowship. We are also grateful to Fahima Faqir and Madison Oger (Institut Curie) for discussions on PCR reactions.

\section{References}

1 Y. Temiz, R. D. Lovchik, G. V. Kaigala and E. Delamarche, Microelectron. Eng., 2015, 132, 156-175.

2 S. Bhattacharya, A. Datta, J. M. Berg and S. Gangopadhyay, J. Microelectromech. Syst., 2005, 14, 590-597.

3 K. Haubert, T. Drier and D. Beebe, Lab Chip, 2006, 6, 1548.

4 E. Ouellet, C. W. T. Yang, T. Lin, L. L. Yang and E. T. Lagally, Langmuir, 2010, 26, 11609-11614.

5 N. Y. Lee and B. H. Chung, Langmuir, 2009, 25, 3861-3866.

6 C. W. Beh, W. Zhou and T.-H. Wang, Lab Chip, 2012, 12, 4120-4127.

7 C. W. Tsao and D. L. DeVoe, Microfluid. Nanofluid., 2009, 6, 1-16.

8 S. Miserere, G. Mottet, V. Taniga, S. Descroix, J.-L. Viovy and L. Malaquin, Lab Chip, 2012, 12, 1849.

9 N. Keller, T. M. Nargang, M. Runck, F. Kotz, A. Striegel, K. Sachsenheimer, D. Klemm, K. Länge, M. Worgull, C. 
Richter, D. Helmer and B. E. Rapp, Lab Chip, 2016, 16, 1561-1564.

10 D. J. Harrison, A. Manz, Z. Fan, H. Luedi and H. M. Widmer, Anal. Chem., 1992, 64, 1926-1932.

11 Z.-J. Jia, Q. Fang and Z.-L. Fang, Anal. Chem., 2004, 76, 5597-5602.

12 S. Waheed, J. M. Cabot, N. P. Macdonald, T. Lewis, R. M. Guijt, B. Paull and M. C. Breadmore, Lab Chip, 2016, 16, 1993-2013.

13 D. Bartolo, G. Degré, P. Nghe and V. Studer, Lab Chip, 2008, 8, 274-279.

14 S. Silvestrini, D. Ferraro, T. Tóth, M. Pierno, T. Carofiglio, G. Mistura and M. Maggini, Lab Chip, 2012, 12, 4041.

15 L. Yu, F. E. H. Tay, G. Xu, B. Chen, M. Avram and C. Iliescu, J. Phys.: Conf. Ser., 2006, 34, 776.

$16 \mathrm{H}$. Wu, B. Huang and R. N. Zare, Lab Chip, 2005, 5, 1393-1398.

17 R. W. R. L. Gajasinghe, S. U. Senveli, S. Rawal, A. Williams, A. Zheng, R. H. Datar, R. J. Cote and O. Tigli, J. Micromech. Microeng., 2014, 24, 75010.

18 L. Tang and N. Y. Lee, Lab Chip, 2010, 10, 1274.

19 P. K. Yuen and V. N. Goral, Lab Chip, 2010, 10, 384-387.

20 J. Saez, L. Basabe-Desmonts and F. Benito-Lopez, Microfluid. Nanofluid., 2016, 20, 116.

21 J. Kim, R. Surapaneni and B. K. Gale, Lab Chip, 2009, 9, 1290.
22 C. S. Thompson and A. R. Abate, Lab Chip, 2013, 13, 632.

23 D. Ferraro, J. Champ, B. Teste, M. Serra, L. Malaquin, J.-L. Viovy, P. de Cremoux and S. Descroix, Sci. Rep., 2016, 6, 25540.

24 P. S. Nunes, P. D. Ohlsson, O. Ordeig and J. P. Kutter, Microfluid. Nanofluid., 2010, 9, 145-161.

25 K. Perez-Toralla, G. Mottet, E. T. Guneri, J. Champ, F.-C. Bidard, J.-Y. Pierga, J. Klijanienko, I. Draskovic, L. Malaquin, J.-L. Viovy and S. Descroix, Lab Chip, 2015, 15, 811-822.

26 K. W. Ro, J. Liu and D. R. Knapp, J. Chromatogr. A, 2006, 1111, 40-47.

27 A. Bruchet, V. Taniga, S. Descroix, L. Malaquin, F. Goutelard and C. Mariet, Talanta, 2013, 116, 488-494.

28 A. Piruska, I. Nikcevic, S. H. Lee, C. Ahn, W. R. Heineman, P. A. Limbach and C. J. Seliskar, Lab Chip, 2005, 5, 1348.

29 E. Piccin, D. Ferraro, P. Sartori, E. Chiarello, M. Pierno and G. Mistura, Sens. Actuators, B, 2014, 196, 525-531.

30 T. Ward, M. Faivre, M. Abkarian and H. A. Stone, Electrophoresis, 2005, 26, 3716-3724.

31 M. T. Guo, A. Rotem, J. A. Heyman and D. A. Weitz, Lab Chip, 2012, 12, 2146.

32 X. Huang, M. J. Gordon and R. N. Zare, Anal. Chem., 1988, 60, 1837-1838.

33 K. Perez-Toralla, J. Champ, M. R. Mohamadi, O. Braun, L. Malaquin, J.-L. Viovy and S. Descroix, Lab Chip, 2013, 13, 4409-4418. 